\title{
PRODUCT INTEGRALS AND EXPONENTIALS IN COMMUTATIVE BANACH ALGEBRAS
}

\author{
JON C. HELTON
}

\begin{abstract}
Functions are from $R \times R$ to $X$, where $R$ represents the real numbers and $X$ represents a commutative Banach algebra with identity element. The function $G \in O C^{\circ}$ on $[a, b]$ only if $a \prod^{b}(1+G)$ exists and is not zero and there exists a subdivision $D$ of $[a, b]$ and a number $B$ such that if $J$ is a refinement of $D$, then $\left[\prod_{J}(1+G)\right]^{-1}$ exists and $\left|\left[\prod_{J}(1+G)\right]^{-1}\right|<B$. If $|G|<1$ on $[a, b]$, then each of the following consists of two equivalent statements: A. (1) $G \in O C^{\circ}$ on $[a, b]$, and (2) $\int_{a}^{b} \ln (1+G)$ exists. B. (1) $G \in O C^{\circ}$ on $[a, b]$ and $\int_{a}^{b}\left|1+G-\prod(1+G)\right|=0$, and (2) $\int_{a}^{b} \mid \ln (1+G)-$ $\int \ln (1+G) \mid=0$. Further, if $\beta>0,|G|<1-\beta$ on $[a, b]$, each of $G\left(p, p^{+}\right), G\left(p^{-}, p\right), G\left(p^{+}, p^{+}\right)$and $G\left(p^{-}, p^{-}\right)$exist for $p \in[a, b]$, $\int_{a}^{b}\left|G^{2}-\int G^{2}\right|=0$ and $G^{2}$ has bounded variation on $[a, b]$, then each of the following consists of two equivalent statements: C. (1) $G \in$ $O C^{\circ}$ on $[a, b]$, and (2) $\int_{a}^{b} G$ exists. D. (1) $G \in 0 C^{\circ}$ on $[a, b]$ and $\int_{a}^{b}\left|1+G-\prod(1+G)\right|=0$, and (2) $\int_{a}^{b}\left|G-\int G\right|=0$.
\end{abstract}

In two recent papers relationships between product integrals and exponentials are investigated for real valued functions. W. P. Davis and J. A. Chatfield [1, Theorem 3, p. 744] show that, if $\int_{a}^{b} G^{2}=0$, then $\int_{a}^{b} G$ exists if and only if $a \prod^{b}(1+G)$ exists and is not zero. Furthermore,

$$
\prod_{a}^{b}(1+G)=\exp \int_{a}^{b} G .
$$

The author [4, Theorem 5] shows that, if $\beta>0,|G|<1-\beta$ on $[a, b]$ and $\int_{a}^{b} G^{2}$ exists, then $\int_{a}^{b} G$ exists if and only if $\prod_{a} \Pi^{b}(1+G)$ exists and is not zero. Furthermore,

$$
\prod_{a}^{b}(1+G)=\exp \int_{a}^{b} \ln (1+G) .
$$

We extend these results to functions with values in a commutative Banach algebra with identity element.

Received by the editors August 1, 1972.

AMS (MOS) subject classifications (1970). Primary 26A39, 26A42, 28A25, 46J99.

Key words and phrases. Sum integral, product integral, subdivision-refinement integral, interval function, interdependency, exponential, commutative Banach algebra.

(C) American Mathematical Society 1973 
Let $X$ be a Banach algebra with identity element. Thus,

(1) $X$ is a complete normed linear space with real or complex scalars,

(2) $X$ is a linear associative algebra with unit 1 , and

(3) if $x, y \in X$, then $|x y| \leqq|x||y|$ and $|1|=1$.

Further, if $x \in X$ and $|x-1|<1$, then

(1) $x^{-1}$ exists and is $\sum_{n=0}^{\infty}(1-x)^{n}$, and

(2) $\left|x^{-1}\right| \leqq[1-|1-x|]^{-1}$.

Exponential and logarithmic functions are defined by the equations

(1) $\exp (x)=\sum_{n=0}^{\infty} x^{n} / n$ ! for $x \in X$, and

(2) $\ln (x)=\sum_{n=1}^{\infty}(-1)^{n-1}(x-1)^{n} / n$ for $x \in X$ and $|x-1|<1$.

These functions are continuous in their domains of definition, and

(1) $\exp [\ln (x)]=x$ if $|x-1|<1$, and

(2) $\ln [\exp (x)]=x$ if $|x-1|<\ln 2$.

In addition, if $x y=y x$, then

(1) $[\exp (x)][\exp (y)]=\exp (x+y)$, and

(2) $\ln (x y)=\ln (x)+\ln (y)$.

If $|x-1|<1,|y-1|<1, x y=y x$ and $z=x y$, then (2) is used to define $\ln (z)$. Consult P. R. Masani [7, pp. 151-152] for additional details and background. Note that $X$ is not the same as the ring $N$ considered by $\mathrm{J}$. S. MacNerney [6, p. 150] and B. W. Helton [2, pp. 298-299].

All integrals and definitions are of the subdivision-refinement type, $R$ denotes the set of all real numbers, and functions are $X$-valued and understood to be defined only on $\{x, y\}$ in $R \times R$ such that $x<y$. The statements that $G$ is bounded, $G \in O B^{\circ}, G \in O P^{\circ}$ and $G \in O U^{\circ}$ on $[a, b]$ mean there exists a subdivision $D$ of $[a, b]$ and a number $B$ such that if $J=\left\{x_{a}\right\}_{0}^{n}$ is a refinement of $D$, then

(1) $|G(u)|<B$ for $u \in J(I)$,

(2) $\sum_{J(I)}|G|<B$,

(3) $\left|\prod_{i}^{j}\left(1+G_{q}\right)\right|<B$ for $1 \leqq i \leqq j \leqq n$, and

(4) $\left[\prod_{i}^{j}\left(1+G_{q}\right)\right]^{-1}$ exists and $\left|\left[\prod_{i}^{j}\left(1+G_{q}\right)\right]^{-1}\right|<B$ for $1 \leqq i \leqq j \leqq n$, respectively, where $G_{q}=G\left(x_{q-1}, x_{q}\right)$ and $J(I)=\left\{\left[x_{q-1}, x_{q}\right]\right\}_{1}^{n}$.

Similarly, statements of the form $G>b$ should be interpreted in terms of subdivisions and refinements. Further, $G \in O C^{\circ}$ on $[a, b]$ only if

(1) $\prod_{a} \Pi^{b}(1+G)$ exists and is not zero, and

(2) there exists a subdivision $D$ of $[a, b]$ and a number $B$ such that if $J$ is a refinement of $D$, then $\left[\prod_{J(I)}(1+G)\right]^{-1}$ exists and $\left|\left[\prod_{J(I)}(1+G)\right]^{-1}\right|<B$.

Also,

(1) $G \in O A^{\circ}$ on $[a, b]$ only if $\int_{a}^{b} G$ exists and $\int_{a}^{b}\left|G-\int G\right|=0$,

(2) $G \in O M^{\circ}$ on $[a, b]$ only if ${ }_{x} \Pi^{y}(1+G)$ exists for $a \leqq x<y \leqq b$ and $\int_{a}^{b}|1+G-\Pi(1+G)|=0$, and

(3) $G \in O L^{\circ}$ on $[a, b]$ only if $\lim _{x \rightarrow p^{+}} G(p, x), \lim _{x \rightarrow p^{-}} G(x, p)$, $\lim _{x, y \rightarrow p^{+}} G(x, y)$ and $\lim _{x, y \rightarrow p^{-}} G(x, y)$ exist for $p \in[a, b]$. 
The statement that the sequence of functions $\left\{S_{n}\right\}_{1}^{\infty}$ converges uniformly to a function $S$ on $[a, b]$ means if $\varepsilon>0$ then there exists a subdivision $D$ of $[a, b]$ and a positive integer $N$ such that if $J$ is a refinement of $D, u \in J(I)$ and $n>N$, then $\left|S_{n}(u)-S(u)\right|<\varepsilon$. See B. W. Helton [2] for more details.

THEOREM 1. Suppose $X$ is commutative. If $G$ is a function from $R \times R$ to $X$ such that $|G|<1$ on $[a, b]$, then the following statements are equivalent:

(1) $G \in O C^{\circ}$ on $[a, b]$, and

(2) $\int_{a}^{b} \ln (1+G)$ exists.

Furthermore, ${ }_{a} \Pi^{b}(1+G)=\exp \int_{a}^{b} \ln (1+G)$.

Theorem 1 follows from the continuity of the exponential and logarithmic functions.

LEMMA 1. If $G$ is a function from $R \times R$ to $X$ such that $|G|<1$ on $[a, b]$ and $G \in O C^{\circ}$ on $[a, b]$, then $G \in O P^{\circ}$ and $O U^{\circ}$ on $[a, b]$.

Lemma 1 follows by the same argument as used in a previous result of the author [5, Theorem 2] since $|G|<1$ implies that $G$ is bounded and that the necessary inverses exist.

Theorem 2. Suppose $X$ is commutative. If $G$ is a function from $R \times R$ to $X$ such that $|G|<1$ on $[a, b]$, then the following statements are equivalent:

(1) $G \in O C^{\circ}$ and $O M^{\circ}$ on $[a, b]$, and

(2) $\ln (1+G) \in O A^{\circ}$ on $[a, b]$.

Furthermore, ${ }_{a} \prod^{b}(1+G)=\exp \int_{a}^{b} \ln (1+G)$.

Proof. (1) $\rightarrow$ (2) It follows from Theorem 1 that $\int_{a}^{b} \ln (1+G)$ exists. Hence, it is only necessary to show that

$$
\int_{a}^{b}\left|\ln (1+G)-\int \ln (1+G)\right|=0 \text {. }
$$

Note that $G \in O P^{\circ}$ and $O U^{\circ}$ on $[a, b]$ [Lemma 1]. Let $\varepsilon>0$. There exists a subdivision $D$ of $[a, b]$ and a number $B$ such that if $J=\left\{x_{q}\right\}_{0}^{n}$ is a refinement of $D$ and $J_{q}$ is a subdivision of $\left[x_{q-1}, x_{q}\right]$ for $1 \leqq q \leqq n$, then

(1) $\left|\left[\prod_{r}^{s}\left(1+G_{q}\right)\right]^{-1}\right|<B$ for $1 \leqq r \leqq s \leqq n$,

(2) $\left|1+\sum_{i=1}^{\infty}(-1)^{i-1}\left\{\left[1+G_{q}\right]\left[\prod_{J q(I)}(1+G)\right]^{-1}-1\right\}^{i-1} / i\right|<B$ for $1 \leqq q \leqq$ $n$,

(3) $\left|\left[1+G_{q}\right]\left[\prod_{J_{q}(I)}(1+G)\right]^{-1}-1\right|<1$ for $1 \leqq q \leqq n$, and

(4) $\sum_{q=1}^{n}\left|1+G_{q}-\prod_{J_{q}(I)}(1+G)\right|<\varepsilon / 2 B^{2}$.

Let $J=\left\{x_{q}\right\}_{0}^{n}$ be a refinement of $D$. For $1 \leqq q \leqq n$, let $J_{q}$ be a subdivision of $\left[x_{q-1}, x_{q}\right]$ such that

$$
\left|\sum_{J_{q}(I)} \ln (1+G)-\int_{x_{q-1}}^{x_{q}} \ln (1+G)\right|<\varepsilon / 2 n .
$$


Thus,

$$
\begin{aligned}
\sum_{q=1}^{n} \mid & \ln \left(1+G_{q}\right)-\int_{x_{q-1}}^{x_{q}} \ln (1+G) \mid \\
\leqq & \sum_{q=1}^{n}\left|\ln \left\{\left[1+G_{q}\right]\left[\prod_{J_{q}(I)}(1+G)\right]^{-1}\left[\prod_{J_{q}(I)}(1+G)\right]\right\}-\sum_{J_{q}(I)} \ln (1+G)\right| \\
& +\sum_{q=1}^{n}\left|\sum_{J_{q}(I)} \ln (1+G)-\int_{x_{q}-1}^{x_{q}} \ln (1+G)\right| \\
< & \sum_{q=1}^{n}\left|\ln \left\{\left[1+G_{q}\right]\left[\prod_{J_{q}(I)}(1+G)\right]^{-1}\right\}\right|+n(\varepsilon / 2 n) \\
= & \sum_{q=1}^{n}\left|\sum_{i=1}^{\infty}(-1)^{i-1}\left\{\left[1+G_{q}\right]\left[\prod_{J_{q}(I)}(1+G)\right]^{-1}-1\right\}^{i} / i\right|+\varepsilon / 2 \\
\leqq & \sum_{q=1}^{n}\left|\left[1+G_{q}\right]\left[\prod_{J_{q}(I)}(1+G)\right]^{-1}-1\right| \\
& \quad\left|1+\sum_{i=1}^{\infty}(-1)^{i-1}\left\{\left[1+G_{q}\right]\left[\prod_{J_{q}(I)}(1+G)\right]^{-1}-1\right\}^{i-1} / i\right|+\varepsilon / 2 \\
\leqq & B \sum_{q=1}^{n}\left|\left[1+G_{q}\right]\left[\prod_{J_{q}(I)}(1+G)\right]^{-1}-1\right|+\varepsilon / 2 \\
\leqq & B \sum_{q=1}^{n}\left|1+G_{q}-\prod_{J_{q}(I)}(1+G)\right|\left|\left[\prod_{J_{q}(I)}(1+G)\right]^{-1}\right|+\varepsilon / 2 \\
\leqq & B^{2} \sum_{q=1}^{n}\left|1+G_{q}-\prod_{J_{q}(I)}(1+G)\right|+\varepsilon / 2 \\
< & B^{2}\left(\varepsilon / 2 B^{2}\right)+\varepsilon / 2=\varepsilon .
\end{aligned}
$$

Therefore, $\ln (1+G) \in O A^{\circ}$ on $[a, b]$.

Proof. (2) $\rightarrow$ (1) It follows from Theorem 1 that

(1) $G \in O C^{\circ}$ on $[a, b]$,

(2) ${ }_{x} \Pi^{y}(1+G)$ exists for $a \leqq x<y \leqq b$, and $\Pi_{a} \Pi^{b}(1+G)=\exp _{a}^{b} \ln (1+G)$.

Hence, it is only necessary to show that

$$
\int_{a}^{b}\left|1+G-\prod(1+G)\right|=0 .
$$

Let $\varepsilon>0$. Lemma 1 implies that $G \in O P^{\circ}$ and $O U^{\circ}$ on $[a, b]$. There exists a subdivision $D$ of $[a, b]$ and a number $B$ such that if $J=\left\{x_{q}\right\}_{0}^{n}$ is a refinement of $D$ and $J_{q}$ is a subdivision of $\left[x_{q-1}, x_{q}\right]$ for $1 \leqq q \leqq n$, then

(1) $\left|\prod_{r}^{s}\left(1+G_{q}\right)\right|<B$ for $1 \leqq r \leqq s \leqq n$,

(2) $\left|\left[1+G_{q}\right]\left[\left.\Gamma\right|_{J^{(I)}}(1+G)\right]^{-1}-1\right|<1$ for $1 \leqq q \leqq n$, 
(3) $\left|\left\{P_{q}\right\}^{-1}\right|<B$ for $1 \leqq q \leqq n$, where

$$
P_{q}=1+\sum_{i=2}^{\infty}(-1)^{i-1}\left\{\left[1+G_{q}\right]\left[\prod_{J_{q}(I)}(1+G)\right]^{-1}-1\right\}^{i-1} / i,
$$

and

(4) $\sum_{q=1}^{n}\left|\ln \left(1+G_{q}\right)-\sum_{J_{q}(I)} \ln (1+G)\right|<\varepsilon / 2 B^{2}$.

Let $J=\left\{x_{q}\right\}_{0}^{n}$ be a refinement of $D$. Further, for $1 \leqq q \leqq n$, let $J_{q}$ be a subdivision of $\left[x_{q-1}, x_{q}\right]$ such that

Thus,

$$
\left|\prod_{J_{q}(I)}(1+G)-{ }_{-x_{q-1}} \prod^{x_{q}}(1+G)\right|<\varepsilon / 2 n .
$$

$$
\begin{aligned}
\sum_{q=1}^{n} \mid 1+G_{q} & -{ }_{-x_{q}-1} \prod^{x_{q}}(1+G) \mid \\
& <\sum_{q=1}^{n}\left|1+G_{q}-\prod_{J_{q}(I)}(1+G)\right|+n(\varepsilon / 2 n) \\
& \leqq \sum_{q=1}^{n}\left|\left[1+G_{q}\right]\left[\prod_{J_{q}(I)}(1+G)\right]^{-1}-1\right|\left|\prod_{J_{q}(I)}(1+G)\right|+\varepsilon / 2 \\
& \leqq B \sum_{q=1}^{n}\left|\left[1+G_{q}\right]\left[\prod_{J_{q}(I)}(1+G)\right]^{-1}-1\right|+\varepsilon / 2 \\
& \leqq B \sum_{q=1}^{n}\left|\left\{\left[1+G_{q}\right]\left[\prod_{J_{q}(I)}(1+G)\right]^{-1}-1\right\}\left\{P_{q}\right\}\right|\left\{P_{q}\right\}^{-1} \mid+\varepsilon / 2 \\
& \leqq B^{2} \sum_{q=1}^{n}\left|\sum_{i=1}^{\infty}(-1)^{i-1}\left\{\left[1+G_{q}\right]\left[\prod_{J_{q}(I)}(1+G)\right]^{-1}-1\right\}^{i} / i\right|+\varepsilon / 2 \\
& =B^{2} \sum_{q=1}^{n}\left|\ln \left\{\left[1+G_{q}\right]\left[\prod_{J_{q}(I)}(1+G)\right]^{-1}\right\}\right|+\varepsilon / 2 \\
& =B^{2} \sum_{q=1}^{n}\left|\ln \left[1+G_{q}\right]-\prod_{J_{q}(I)} \ln (1+G)\right|+\varepsilon / 2 \\
& <B^{2}\left(\varepsilon / 2 B^{2}\right)+\varepsilon / 2=\varepsilon
\end{aligned}
$$

Therefore, $G \in O M^{\circ}$ on $[a, b]$.

LEMMA 2. If $G$ is a function from $R \times R$ to $X, G \in O B^{\circ}$ on $[a, b],\left\{S_{n}\right\}_{1}^{\infty}$ is a sequence of functions from $R \times R$ to $X$ converging uniformly to a bounded function $S$ on $[a, b]$ and $S_{n} G \in O A^{\circ}$ on $[a, b]$ for $n=1,2, \cdots$, then $S G \in O A^{\circ}$ on $[a, b], \lim _{n \rightarrow \infty} \int_{a}^{b} S_{n} G$ exists and $\int_{a}^{b} S G=\lim _{n \rightarrow \infty} \int_{a}^{b} S_{n} G$.

The proof of Lemma 2 is straightforward and, therefore, we omit it. 
LEMMA 3. If $H$ and $G$ are functions from $R \times R$ to $X, H \in 0 L^{\circ}$ on $[a, b]$ and $G \in O A^{\circ}$ and $O B^{\circ}$ on $[a, b]$, then $H G \in O A^{\circ}$ and $O M^{\circ}$ on $[a, b][3$, Theorem 2, p. 494].

LeMma 4. If $\beta>0$ and $G$ is a function from $R \times R$ to $X$ such that $|G|<$ $1-\beta$ on $[a, b], G \in O L^{\circ}$ on $[a, b]$ and $G^{2} \in O A^{\circ}$ and $O B^{\circ}$ on $[a, b]$, then

is in $O A^{\circ}$ on $[a, b]$.

$$
\sum_{n=2}^{\infty}(-1)^{n-1} G^{n} / n
$$

Proof. Since $G \in O L^{\circ}$ on $[a, b]$,

$$
\sum_{n=2}^{p}(-1)^{n-1}\left(G^{n-2}\right) / n
$$

is in $O L^{\circ}$ on $[a, b]$ for $p=2,3,4, \cdots$. Thus,

$$
\left[\sum_{n=2}^{p}(-1)^{n-1}\left(G^{n-2}\right) / n\right] G^{2}
$$

is in $O A^{\circ}$ on $[a, b]$ for $p=2,3,4, \cdots$ [Lemma 3]. Therefore, since

converges uniformly to

$$
\left\{\sum_{n=2}^{p}(-1)^{n-1}\left(G^{n-2}\right) / n\right\}_{p=2}^{\infty}
$$

$$
\sum_{n=2}^{\infty}(-1)^{n-1}\left(G^{n-2}\right) / n
$$

on $[a, b]$, it follows from Lemma 2 that

$$
\left[\sum_{n=2}^{\infty}(-1)^{n-1}\left(G^{n-2}\right) / n\right] G^{2}=\sum_{n=2}^{\infty}(-1)^{n-1} G^{n} / n
$$

is in $O A^{\circ}$ on $[a, b]$.

We note that a similar justification of Lemma 4 for real valued functions is contained in the proof of Theorem $5(1 \rightarrow 2)$ of a previous paper by the author [4].

THEOREM 3. Suppose $X$ is commutative. If $\beta>0$ and $G$ is a function from $R \times R$ to $X$ such that $|G|<1-\beta$ on $[a, b], G \in O L^{\circ}$ on $[a, b]$ and $G^{2} \in O A^{\circ}$ and $O B^{\circ}$ on $[a, b]$, then the following statements are equivalent:

(1) $G \in O C^{\circ}$ on $[a, b]$, and

(2) $\int_{a}^{b} G$ exists.

Furthermore, ${ }_{a} \Pi^{b}(1+G)=\exp \int_{a}^{b} \ln (1+G)$. 
Proof. It follows from Lemma 4 that

$$
\int_{a}^{b} \sum_{n=2}^{\infty}(-1)^{n-1} G^{n} / n
$$

exists. Hence, if (1) is true, then $\int_{a}^{b} G$ exists since Theorem 1 implies that

$$
\int_{a}^{b} \ln (1+G)=\int_{a}^{b} \sum_{n=1}^{\infty}(-1)^{n-1} G^{n} / n
$$

exists. Also, if (2) is true, then Theorem 1 implies that (1) is true since

$$
\int_{a}^{b} \sum_{n=1}^{\infty}(-1)^{n-1} G^{n} / n=\int_{a}^{b} \ln (1+G)
$$

exists. In addition, Theorem 1 also implies that

$$
\prod_{a}^{b}(1+G)=\exp \int_{a}^{b} \ln (1+G) \text {. }
$$

THEOREM 4. Suppose $X$ is commutative. If $\beta>0$ and $G$ is a function from $R \times R$ to $X$ such that $|G|<1-\beta$ on $[a, b], G \in O L^{\circ}$ on $[a, b]$ and $G^{2} \in O A^{\circ}$ and $O B^{\circ}$ on $[a, b]$, then the following statements are equivalent:

(1) $G \in O C^{\circ}$ and $O M^{\circ}$ on $[a, b]$, and

(2) $G \in O A^{\circ}$ on $[a, b]$.

Furthermore, ${ }_{a} \Pi^{b}(1+G)=\exp \int_{a}^{b} \ln (1+G)$.

Proof. It follows from Lemma 4 that

$$
\sum_{n=2}^{\infty}(-1)^{n-1} G^{n} / n
$$

is in $O A^{\circ}$ on $[a, b]$. Hence, if (1) is true, then $G \in O A^{\circ}$ on $[a, b]$ since Theorem 2 implies that

$$
\ln (1+G)=\sum_{n=2}^{\infty}(-1)^{n-1} G^{n} / n
$$

is in $O A^{\circ}$ on $[a, b]$. Also, if (2) is true, then Theorem 2 implies that (1) is true since

$$
\sum_{n=1}^{\infty}(-1)^{n-1} G^{n} / n=\ln (1+G)
$$

is in $O A^{\circ}$ on $[a, b]$. In addition, Theorem 2 also implies that

$$
\prod^{b}(1+G)=\exp \int_{a}^{b} \ln (1+G) .
$$




\section{BIBLIOGRAPHY}

1. W. P. Davis and J. A. Chatfield, Concerning product integrals and exponentials, Proc. Amer. Math. Soc. 25 (1970), 743-747. MR 42 \#1970.

2. B. W. Helton, Integral equations and product integrals, Pacific J. Math. 16 (1966), 297-322. MR 32 \#6167.

3. - A product integral representation for a Gronwall inequality, Proc. Amer. Math. Soc. 23 (1969), 493-500. MR 40 \#1562.

4. J. C. Helton, Some interdependencies of sum and product integrals, Proc. Amer. Math. Soc. 37 (1973), 201-206.

5. — Product integrals, bounds and inverses, Texas J. Sci. (to appear).

6. J. S. MacNerney, Integral equations and semigroups, Illinois J. Math. 7 (1963), 148-173. MR 26 \#1726.

7. P. R. Masani, Multiplicative Riemann integration in normed rings, Trans. Amer. Math. Soc. 61 (1947), 147-192. MR 8, 321.

Department of Mathematics, Arizona State University, Tempe, Arizona 85281 